\title{
Free Recall Episodic Memory Performance Predicts Dementia Ten Years prior to Clinical Diagnosis: Findings from the Betula Longitudinal Study
}

\author{
Carl-Johan Boraxbekk $^{a} \quad$ Anders Lundquist $^{b} \quad$ Annelie Nordin ${ }^{\mathrm{e}}$ \\ Lars Nyberg ${ }^{c, d}$ Lars-Göran Nilsson ${ }^{f}$ Rolf Adolfsson ${ }^{e}$ \\ ${ }^{a}$ CEDAR - Center for Demographic and Aging Research, and ${ }^{b}$ Department of Statistics, \\ 'Division of Physiology, Department of Integrative Medical Biology, ${ }^{\mathrm{d}}$ Division of Diagnostic \\ Radiology, Department of Radiation Sciences, and ${ }^{~}$ Division of Psychiatry, Department of \\ Clinical Sciences, Umeå University, Umeå, and ${ }^{\dagger}$ Aging Research Center, Karolinska Institutet, \\ Stockholm, Sweden
}

Key Words

Episodic memory · Cognitive markers · Dementia - Predictive validity

\begin{abstract}
Background/Aims: Early dementia diagnosis is a considerable challenge. The present study examined the predictive value of cognitive performance for a future clinical diagnosis of lateonset Alzheimer's disease or vascular dementia in a random population sample. Methods: Cognitive performance was retrospectively compared between three groups of participants from the Betula longitudinal cohort. Group 1 developed dementia 11-22 years after baseline testing $(n=111)$ and group 2 after $1-10$ years $(n=280)$; group 3 showed no deterioration towards dementia during the study period ( $n=2,855)$. Multinomial logistic regression analysis was used to investigate the predictive value of tests reflecting episodic memory performance, semantic memory performance, visuospatial ability, and prospective memory performance. Results: Age- and education-corrected performance on two free recall episodic memory tests significantly predicted dementia 10 years prior to clinical diagnosis. Free recall performance also predicted dementia 11-22 years prior to diagnosis when controlling for education, but not when age was added to the model. Conclusion: The present results support the suggestion that two free recall-based tests of episodic memory function may be useful for detecting individuals at risk of developing dementia 10 years prior to clinical diagnosis.
\end{abstract}




\section{Introduction}

Advances in treatments and the possible prevention of dementia onset increase the importance of early diagnosis. Memory failure is one of the earliest indications of dementia, suggesting that neuropsychological assessment may be an important diagnostic tool [1]. Several studies show that loss of episodic memory functions is a core feature of the entire dementing process [2-5]. Research on early signs of dementia have proposed that tests reflecting episodic memory performance could potentially distinguish between normal aging and a progression towards dementia up to 6 years prior to dementia onset [3]. Findings further indicate that tests utilizing a free recall-based assessment appear to be the most effective in early disease stages [6]. The dementing process also involves other cognitive functions, including semantic memory - e.g. fluency, verbal ability, and visuospatial ability $[2,7]$. However, the tests of these functions have a weaker predictive value than episodic memory tests $[8,9]$.

Notably, the most common dementia disorders - Alzheimer's disease (AD) and vascular dementia (VaD) - seem to impactperformance equally on cognitive tests. For example, Fahlander et al. [10] showed that patients with $\mathrm{AD}$ and those with $\mathrm{VaD}$ did not differ in their performance on tests reflecting episodic memory, semantic memory, and visuospatial ability $[11,12]$. Key remaining questions include how early a forthcoming clinical diagnosis of dementia can be predicted based on cognitive performance, and which tests are the most sensitive.

The present study aimed to investigate early cognitive signs of a future dementia diagnosis. Using data from a random population sample, we investigated the possibility of using performance on cognitive tests to differentiate between normal aging and signs of dementia development 10 or even 20 years prior to a clinical dementia diagnosis. A second aim of our study was to investigate potential differences in cognitive test performance between individuals who would be diagnosed with AD and those who would be diagnosed with VaD. Based on previous results [10], we did not expect to find a significant difference. Neuropsychological tests likely show differing sensitivities in predicting a dementia diagnosis, especially with varying time between testing and diagnosis $[5,6,8]$. We hypothesized that the earliest signs of upcoming dementia would be apparent in the performance of free recall rather than cued recall tests. Moreover, the duration between testing and dementia diagnosis can influence test performances reflecting other cognitive domains, e.g. fluency and visuospatial ability [6, 8]. We hypothesized that performance on a broader range of cognitive tests would have the potency to discriminate participants at risk of dementia closer to the clinical diagnosis.

\section{Subjects and Methods}

\section{Participants and Clinical Diagnosis of Dementia}

The Betula Project is an ongoing population-based prospective study that was started in 1988 with the general aim of investigating the development of memory functions and health across the adult lifespan and the specific aim of exploring risk factors and early signs of dementia, including cognitive data patterns. The cohort is described in detail by Nilsson and colleagues [13, 14]. Briefly, 6 samples of participants (S1-S6) were randomly recruited from the population of a community of about 100,000 inhabitants in northern Sweden, representing a culturally and ethnically homogenous sample. Since the initial testing occasion, participants have been reexamined in 5-year intervals. Each test wave (T1: 1988-1990; T2: 1993-1995; T3: 1998-2001; T4: 2003-2005; T5: 2008-2010) included health examinations, extensive cognitive testing, and a careful evaluation of the current status with regard to dementia. Altogether, this protocol provided data enabling the study of early cognitive signs up to 20 years prior to a clinical dementia diagnosis - the longest length of time studied to date. 
Boraxbekk et al.: Free Recall Episodic Memory Performance Predicts Dementia Ten

Years prior to Clinical Diagnosis: Findings from the Betula Longitudinal Study

Table 1. Characteristics of the participants

\begin{tabular}{lllll}
\hline & Controls & Dementia 1-10 & Dementia 11-22 & Statistics \\
\hline Subjects, $\mathrm{n}$ & 2,855 & 280 & 111 & \\
AD, $\mathrm{n}$ & & 188 & 56 & \\
VaD, $\mathrm{n}$ & & 92 & 55 & \\
\hline Mean age at inclusion (range), years & $57.3(35-90)$ & $74.6(40-90)$ & $66.1(35-80)$ & $\mathrm{F}_{2,2,853}=230.9, \mathrm{p}<0.001$ \\
Mean age at dementia diagnosis (range), years & & $80.5(47-94)$ & $80.5(57-95)$ & $\mathrm{F}_{1,30}=0.067, \mathrm{p}=0.80$ \\
Mean time from initial testing to dementia & & & & \\
$\quad$ diagnosis, years & & 5.6 & 14.2 & $\mathrm{~F}_{1,30}=850.1, \mathrm{p}<0.001$ \\
Male, \% & 47.2 & 33.5 & 38.4 & $\chi^{2}(2)=17.5, \mathrm{p}<0.001$ \\
Female, \% & 52.8 & 66.5 & 61.6 & \\
Mean education (SD), years & $10.4(4.3)$ & $7.7(3.0)$ & $7.9(2.8)$ & $\mathrm{F}_{2,2,853}=71.2, \mathrm{p}<0.001$ \\
APOE \&4, $\mathrm{n}(\%)$ & $681(22)$ & $111(39)$ & $42(38)$ & $\chi^{2}(2)=43.0, \mathrm{p}<0.001$ \\
\hline
\end{tabular}

For the present study, we utilized data from Betula study cohorts S1, S2, S3, and S4, including cognitive measures obtained at the baseline testing occasion (T1, T2, or T3). We included participants who developed dementia (AD or VaD) at least 1 year after their initial testing. As controls, we included participants who did not develop dementia during the study period and who were part of the same cohorts and testing occasions as the included individuals with dementia. The included individuals were categorized into three subgroups based on dementia status and time elapsed until dementia diagnosis: group 1 (dementia 11-22), i.e. individuals diagnosed with dementia 11-22 years after inclusion ( $\mathrm{n}=111$ ); group 2 (dementia 1-10), i.e. individuals diagnosed with dementia 1-10 years after inclusion $(n=280)$, and group 3, i.e. controls $(n=2,855)$. Table 1 shows the participants' characteristics.

The dementia diagnosis was based on established core criteria that imply impairment in several cognitive domains - such as memory, language, reasoning, personality, and visuospatial abilities - reducing functional capacity and interfering with activities of daily living [15]. The diagnostic procedure adopted a conservative approach using a combination of predetermined clinical signs according to international guidelines. AD and VaD were diagnosed in accordance with the DSM-IV criteria [15]. However, the clinical picture and the course of the disease were decisive for the diagnostic distinction. The dementia subtypes AD and $\mathrm{VaD}$ were not diagnosed unless there was a dominating progressive nature of the course. Age at onset was defined retrospectively based on when the disorder first manifested to the extent that the severity of cognitive impairment was sufficient to adversely affect independent functioning consistent with dementia.

Dementia diagnosis in the Betula study has previously been described [16]. During the study period (T1-T5; 1988-2010) retrospective analyses of clinical signs and symptoms were performed by evaluations of medical records providing information on all the medical consultations and examinations conducted. Results obtained by neuroimaging, PET, CSF biomarkers, or confirmation at autopsy were taken into account when such information was available in the medical records. The procedure was applied to all participants at baseline and every 5 th year to identify early symptoms, cognitive and functional performance over time, and the year in which the individual fulfilled the overall criteria for dementia. A research geropsychiatrist coordinated the diagnostic evaluation and was responsible for the final diagnoses throughout the study period, ranging from inclusion up to T5 or, for the deceased participants, up to the date of death. At test waves T1, T2, and T3, up to 4 additional geropsychiatrists participated in the evaluation process, thereby establishing a solid ground for a diagnostic consensus. The research geropsychiatrist had access to all investigational materials available in the medical records. 
In addition to the medical record screening, the study participants were interviewed for 2-3 $\mathrm{h}$ by an experienced research nurse and also tested cognitively approximately 1 week afterwards by a cognitive test leader for another 2-3 h. The observations made at the health and cognitive evaluations formed the basis for a decision on performing a more extended diagnostic evaluation. If a participant scored $<24$ points on the MMSE or dropped in cognitive performance from high to normal/low or from normal to low compared with the previous examination, he or she was considered at higher risk than those participants passing without any remark. Also, if a participant expressed subjective memory impairment and if the research staff objectively noticed confusion or other cognitive loss of function, participants were considered at risk. Participants at risk were subjected to a careful evaluation and, in some cases, to an extended clinical examination for further evaluation of the cause of the changes in behavior or performance.

At T5, an extensive quality assurance of the dementia diagnosis was performed in that all medical records of those with an established dementia diagnosis were blindly reevaluated with regard to consistency of dementia type and age at onset. A comparison between the previously diagnosed subtypes and the newly proposed ones showed that approximately 95\% were congruent with the previously given diagnosis. In those cases where the diagnoses differed, it was evident that the reevaluated diagnoses at $\mathrm{T} 5$ were more reliable, probably due to the longer follow-up time. The diagnostic evaluation at T5 showed that 444 of a total of 4,069 individuals from Betula cohorts S1-S5 had developed a dementia disorder, with a clear preponderance of $\mathrm{AD}(55 \%)$ and $\mathrm{VaD}(34 \%)$. Participants with frontal lobe dementia $(0.7 \%)$, Lewy body dementia (1.8\%), or Parkinson's dementia (2.5\%) were diagnosed on basis of extensive examinations performed by the treating neurologist. Participants that did not fulfil the criteria for either $\mathrm{AD}$ or $\mathrm{VaD}$ or any of the other subtypes received the diagnosis 'dementia not otherwise specified' (3.4\%). However, these subtypes were outside the scope of the current study. Furthermore, the reevaluation showed that only $19(0.4 \%)$ of all 4,445 Betula participants (S1-S5) were found to be improperly included in the study (dementia in progress at inclusion), which provides a strong support for the diagnostic evaluation method used.

\section{Neuropsychological Tests}

In the Betula cohort, the memory test battery was administered by trained psychometricians. On each testing occasion, the duration of the testing session was $1.5-2 \mathrm{~h}$ for each participant. In most instances, memory testing and health examinations occurred in dedicated rooms at the Department of Psychology, Umeå University. However, a small number of elderly individuals were unable to visit the laboratory for various reasons and were therefore tested in their homes. For a test to be included in the Betula study, it had to meet the major criteria of being rooted in extant theories of memory and being sensitive to various memory deficits in old age. Most of the tests utilized were designed especially for the Betula study and were thoroughly tested in many previous experiments, demonstrating moderate-to-high reliabilities and stability coefficients $[17,18]$. The present study included only tests that were administered in exactly the same manner across testing occasions. The subtests characterized below were used in the present study in the order described. The complete list of available tests and detailed descriptions of the testing procedures are reported by Nilsson and colleagues $[13,14,19]$.

Prospective Memory

The first testadministered was an instruction regarding the subsequent test of prospective memory (PROSP). Participants were instructed to remind the experimenter at the end of the session to sign a piece of paper. This test was an event-based prospective memory task, which was included in the Betula study based on previous findings of decreasing performance as a function of age [20]. 
Boraxbekk et al.: Free Recall Episodic Memory Performance Predicts Dementia Ten

Years prior to Clinical Diagnosis: Findings from the Betula Longitudinal Study

Episodic Memory: Sentence Learning with and without Encoding Enactment

The participants were asked to learn two lists of 16 short sentences (e.g. 'lift the pen'). Each sentence was visually presented on an index card for $8 \mathrm{~s}$. For one of the lists, the participants were asked to enact each presented sentence, whereas no enactment was required for the other list [21]. Each list presentation was immediately followed by administration of a free recall test. These tasks are referred to as free recall of sentences with enactment (SPTB) and without enactment (VTB). The second free recall test was followed by a cued recall test of the nouns presented in each of the two lists [22]. This provided us with two additional measures of episodic memory performance: cued recall for sentences that were initially learned with enactment (SPTCRC) and for sentences initially learned without enactment (VTCRC).

Word Fluency: Semantic Memory with Time Requirements

First, the participants were asked to generate as many words as possible starting with the letter ' $\mathrm{A}$ ' in 1 min (FLUA). They were then asked to generate as many 5-letter words as possible that started with the initial letter ' $M$ ' in 1 min (FLU5M). In the last fluency task, the participants were asked to list as many professions starting with the letter ' $\mathrm{B}$ ' as possible in 1 min (FLUPB) [23].

Visuospatial Memory: Block Design

This test (BLOCK) is a subtest of the Wechsler Adult Intelligence Scale (WAIS) [24], which shows age-related differences in performance [25]. The participants were asked to position red and white blocks such that the blocks matched a pattern shown on a picture (maximum of 9 designs).

\section{Prospective Memory}

This test (PROSP) was administered to assess whether participants remembered to remind the experimenter about the task given at the beginning of the testing session. If they remembered it without any cue, they received 4 points for prospective memory. If they failed to remind the experimenter, they were cued regarding this task in two steps [20]. Two tests were administered by the nurse at the health examination.

Vocabulary, Semantic Memory

The participants were given a list of 30 target words, together with an additional 5 words for each target word. The task was to indicate which of the 5 words were synonymous with the target word (SRB) [26].

Mini-Mental State Examination

This series of tests was followed by the MMSE [27], the final test used in the present study.

\section{Statistical Analysis}

To compare the mean scores on the various tests across groups - and to thereby assess which tests seemed suitable for subsequent classification/discrimination models - we performed one-way ANOVAs for each cognitive test, using the test score as the outcome variable and the dementia group as the factor. For each outcome variable, the normal distribution was visually inspected, and Levene's test of homogeneity of variances across groups was performed. Depending on the outcome, this test was followed by a parametric or a nonparametric ANOVA (Kruskal-Wallis). In the event of finding no overall group difference with respect to the mean score, the test in question was considered to likely be ill-suited for 
Boraxbekk et al.: Free Recall Episodic Memory Performance Predicts Dementia Ten

our classification purpose. To reduce multiple testing issues, we used the Bonferroni correction when examining differences across groups.

The present study employed multinomial logistic regression analysis to investigate whether single-time-point cognitive data could be used to predict dementia up to 10 years or up to 22 years prior to clinical diagnosis. Such analysis enabled the determination of which, if any, of the selected cognitive tests significantly influenced the likelihood of being included in any of the dementia groups. The dependent variables were the three groups, with the nondemented group selected as the reference group, and the cognitive tests were used as the predictor variables. All tests were added simultaneously to the model. Previous studies have reported that low education is a strong predictor of developing dementia [28, 29]; thus, we immediately controlled for educational level by adding this variable to the model. We also controlled for age. Our final analysis aimed to determine whether cognitive testing could be used to discriminate between the dementia subtypes (AD and VaD) using ANOVAs on each cognitive test, with dementia subtype as the factor. All analyses were performed using SPSS 19 software with a significance level of $\mathrm{p}<0.05$.

\section{Results}

\section{Analysis of Variance}

All variables were normally distributed. The results obtained from Levene's test suggested that the SPTB, VTB, VTCRC, MMSE, SRB, and BLOCK tests did not satisfy the assumption of variance homogeneity across groups; therefore, these tests were analyzed using the KruskalWallis procedure. The remaining tests were subjected to a standard parametric ANOVA. For all the tests examined, the mean unadjusted scores at inclusion in the Betula study showed significant between-group differences $(\mathrm{p}<0.001)$, suggesting that all tests could be considered for subsequent classification/discrimination modeling (see table 1). Furthermore, post hoc testing revealed that in the SPTB, SPTCRC, VTB, VTCRC, BLOCK, and PROSP tests, the controls performed significantly better than the other groups, and group 1 (dementia 11-22) performed better than group 2 (dementia 1-10). For the SRB, FLUPB, and MMSE tests, performance did not differ between the controls and group 1 (dementia 11-22), but both the controls and group 1 performed better than group 2 (dementia 1-10). For the FLUA and FLU5M tests, the controls performed better than the two other groups, with no differences between group 1 (dementia 11-22) and group 2 (dementia 1-10) (table 2). Regarding the dementia subtypes, none of the cognitive tests showed significant differences between AD and VaD (all p > 0.05) (table 3).

\section{Multinomial Logistic Regression Analysis}

The results of the backward elimination step indicated that the cued recall tests (VTCRC and SPTCRC) and two of the fluency tests (FLUPB and FLUA) had no significant overall influence in the model or on inclusion in the dementia groups. Although the mean scores on these tests significantly differed between the three groups, the tests could not be used to predict dementia onset. These tests were thus removed from the model.

Analyses controlling for education showed that the following tests were significant for determining inclusion in group 1 (dementia 11-22): free recall of enacted sentences (SPTB; estimated $\mathrm{OR}=0.92, \mathrm{p}=0.039,95 \% \mathrm{CI}: 0.84-0.99$ ) and vocabulary (SRB; estimated $\mathrm{OR}=1.06$, $\mathrm{p}=0.020,95 \% \mathrm{CI}: 1.01-1.11)$. For SRB, the OR was in an unexpected direction - that is, when fitting a model with all the variables included, a higher score on SRB increased the odds of inclusion in the dementia group. This was surprising, since the mean SRB score in the dementia group was lower than in the control group. When a model was fit with only SRB, it failed to 
Boraxbekk et al.: Free Recall Episodic Memory Performance Predicts Dementia Ten

Years prior to Clinical Diagnosis: Findings from the Betula Longitudinal Study

Table 2. Unadjusted cognitive performance across groups

\begin{tabular}{lcccl}
\hline Variable & Controls & Dementia $1-10$ & Dementia $11-22$ & ANOVA \\
\hline SPTB & $8.5(3.2)$ & $5.3(2.8)$ & $7.3(2.8)$ & $\mathrm{F}_{2,2,852}=158.6, \mathrm{p}<0.001$ \\
SPTCRC & $9.9(2.8)$ & $7.2(2.9)$ & $8.9(2.5)$ & $\mathrm{F}_{2,2,850}=127.6, \mathrm{p}<0.001$ \\
VTB & $4.8(2.7)$ & $2.6(1.9)$ & $4.2(2.3)$ & $\mathrm{F}_{2,2,854}=92.4, \mathrm{p}<0.001$ \\
VTCRC & $6.1(3.3)$ & $3.9(2.7)$ & $5.3(3.1)$ & $\mathrm{F}_{2,2,853}=74.7, \mathrm{p}<0.001$ \\
SRB & $21.7(5.3)$ & $18.7(6.4)$ & $21.1(5.0)$ & $\mathrm{F}_{2,2,848}=54.3, \mathrm{p}<0.001$ \\
FLUA & $11.1(4.8)$ & $8.5(4.5)$ & $9.5(4.5)$ & $\mathrm{F}_{2,2,852}=44.5, \mathrm{p}<0.001$ \\
FLU5M & $5.4(3.1)$ & $4.3(2.9)$ & $4.9(3.0)$ & $\mathrm{F}_{2,2,850}=20.3, \mathrm{p}<0.001$ \\
FLUPB & $4.6(2.2)$ & $3.5(2.1)$ & $4.2(2.1)$ & $\mathrm{F}_{2,2,852}=38.1, \mathrm{p}<0.001$ \\
BLOCK & $26.5(10.9)$ & $17(8.3)$ & $23.4(8.3)$ & $\mathrm{F}_{2,2,851}=114.6, \mathrm{p}<0.001$ \\
PROSP & $3.0(1.1)$ & $2.3(1.1)$ & $2.7(1.1)$ & $\mathrm{F}_{2,2,847}=62.3, \mathrm{p}<0.001$ \\
MMSE & $27.8(1.9)$ & $26.3(2.3)$ & $27.7(1.7)$ & $\mathrm{F}_{2,2,852}=78.2, \mathrm{p}<0.001$ \\
\hline
\end{tabular}

Values are means (SD). Data represent ANOVA results over controls, group 2 (dementia 1-10), and group 1 (dementia 11-22). Bonferroni post hoc analyses revealed that for SPTB, SPTCRC, VTB, VTCRC, BLOCK, and PROSP there were significant $(\mathrm{p}<0.05)$ differences between all groups. For SRB, FLUPB, and the MMSE, group 2 (dementia 1-10) performed significantly higher than group 1 (dementia 11-22). For FLUA and FLU5M, the controls performed significantly higher than dementia group 2 (dementia 1-10) and group 1 (dementia 11-22).

Table 3. Performance on cognitive tests presented separately for participants diagnosed with $\mathrm{AD}$ and $\mathrm{VaD}$

\begin{tabular}{lrrl}
\hline Variable & \multicolumn{1}{l}{ AD } & \multicolumn{1}{l}{ VaD } & \multicolumn{1}{l}{ ANOVA } \\
\hline SPTB & $5.8(2.9)$ & $5.8(3.0)$ & $\mathrm{F}_{1,389}=0.001, \mathrm{p}=0.9$ \\
SPTCRC & $7.7(2.9)$ & $7.6(2.9)$ & $\mathrm{F}_{1,389}=0.2, \mathrm{p}=0.7$ \\
VTB & $3.1(2.2)$ & $3.1(2.0)$ & $\mathrm{F}_{1,389}=0.04, \mathrm{p}=0.8$ \\
VTCRC & $4.5(3.0)$ & $3.9(2.8)$ & $\mathrm{F}_{1,389}=3.2, \mathrm{p}=0.08$ \\
SRB & $19.3(6.0)$ & $19.3(6.4)$ & $\mathrm{F}_{1,389}=0.001, \mathrm{p}=0.9$ \\
FLUA & $8.9(4.5)$ & $8.6(4.6)$ & $\mathrm{F}_{1,389}=0.3, \mathrm{p}=0.6$ \\
FLU5M & $4.5(2.9)$ & $4.3(2.9)$ & $\mathrm{F}_{1,389}=0.7, \mathrm{p}=0.4$ \\
FLUPB & $3.8(2.2)$ & $3.6(2.0)$ & $\mathrm{F}_{1,389}=0.7, \mathrm{p}=0.4$ \\
BLOCK & $18.4(8.4)$ & $19.4(9.3)$ & $\mathrm{F}_{1,389}=1.2, \mathrm{p}=0.3$ \\
PROSP & $2.4(1.1)$ & $2.4(1.1)$ & $\mathrm{F}_{1,389}=0.2, \mathrm{p}=0.7$ \\
MMSE & $26.7(2.2)$ & $26.8(2.3)$ & $\mathrm{F}_{1,389}=0.3, \mathrm{p}=0.6$ \\
\hline
\end{tabular}

Values are means (SD). Participants diagnosed with AD and those diagnosed with VaD showed no significant differences in performance on any of the cognitive tests.

reach significance $(\mathrm{p}=0.275)$. Thus, SRB was unable to predict dementia onset in group 1 (dementia 11-22). SPTB also failed to predict dementia in group 1 when age was added as a covariate.

Analyses controlling for education showed that the following tests were significant for determining clinical dementia diagnosis within 1-10 years: free recall of both enacted sentences (SPTB; estimated OR $=0.83, \mathrm{p}<0.0005,95 \% \mathrm{CI}: 0.78-0.88$ ) and not enacted sentences (VTB; estimated OR $=0.85, \mathrm{p}<0.0005,95 \%$ CI: $0.79-0.92$ ), prospective memory (PROSP; estimated OR $=0.80, \mathrm{p}<0.0005,95 \%$ CI: 0.70-0.90), fluency (FLU5M; estimated $\mathrm{OR}=1.06, \mathrm{p}=0.023,95 \% \mathrm{CI}: 1.01-1.12)$, visuospatial memory (BLOCK; estimated OR = 0.96, p < 0.0005, 95\% CI: 0.94-0.98), MMSE results (estimated OR $=0.92, p=0.030,95 \%$ CI: 0.86-0.99), and vocabulary (SRB; estimated OR $=1.05, p=0.001,95 \%$ CI: $1.02-1.09$ ). 


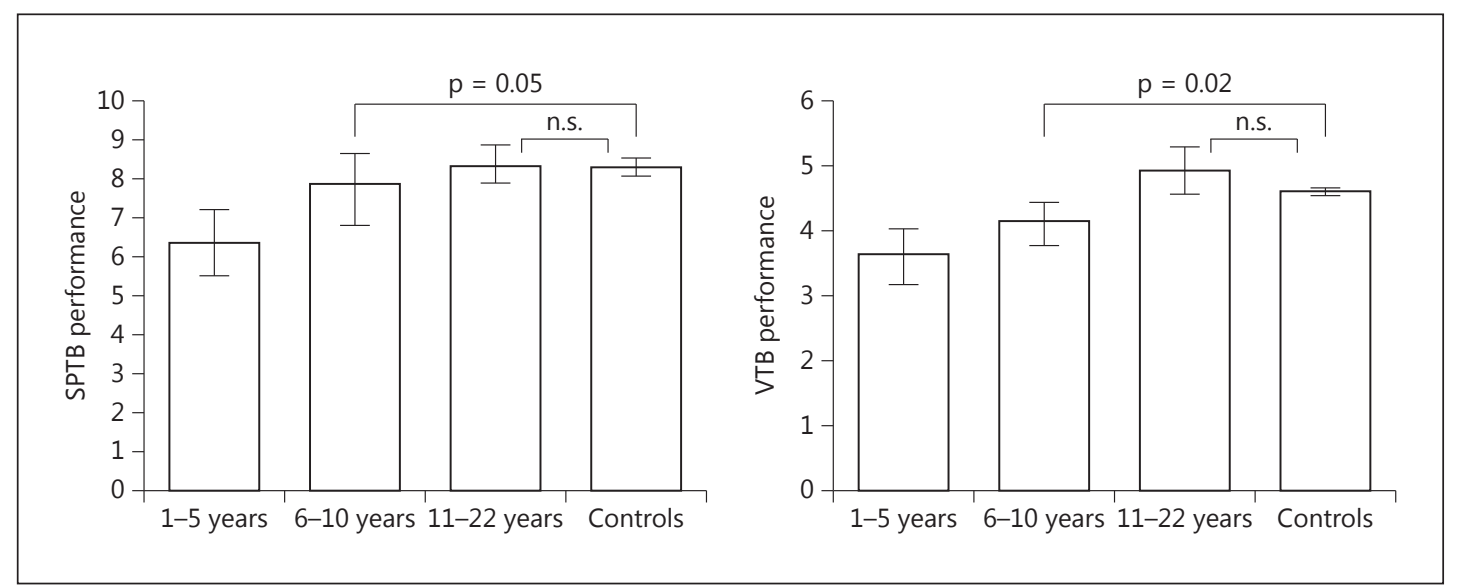

Fig. 1. Age- and education-corrected values from two free recall episodic memory tests (SPTB and VTB) were significantly lower 6-10 years and 1-5 years before dementia diagnosis. There was no significant difference between controls and those individuals that would be diagnosed with dementia within 11-22 years. Bars indicate 95\% CI. n.s. = Nonsignificant.

The McFadden pseudo- $\mathrm{R}^{2}$ for this model was 0.14 . Once again, SRB showed an unexpected direction, such that a higher score increased the odds of inclusion in the dementia group. The FLU5M fluency test also showed this direction. However, fitting a model with only FLU5M or SRB yielded the expected results for both SRB (estimated OR $=0.92, \mathrm{p}<0.0005$, 95\% CI: 0.90-0.93) and FLU5M (estimated OR $=0.89, \mathrm{p}<0.0005,95 \%$ CI: 0.85-0.93) - in other words, a higher score significantly decreased the odds of developing dementia within 10 years from testing.

Notably, education failed to predict inclusion in group 2 (dementia 1-10) $(\mathrm{p}=0.075)$. With age added as a covariate, the following tests remained significant: episodic free recall of enacted sentences (SPTB; estimated OR $=0.90, p=0.001,95 \% \mathrm{CI}: 0.85-0.96$ ), episodic free recall of sentences without enactment (VTB; estimated OR $=0.90, p=0.011,95 \%$ CI: $0.83-$ 0.98), and prospective memory (PROSP; estimated OR $=0.87, \mathrm{p}=0.042,95 \% \mathrm{CI}: 0.77-0.99$ ). The McFadden pseudo- $\mathrm{R}^{2}$ for this model was 0.17 . Thus, SPTB, VTB, and PROSP showed good predictive values up to 10 years before clinical dementia diagnosis, even after controlling for education and age.

\section{Control Analysis}

Since low education and old age are strong predictors of future dementia, we further investigated how education- and age-corrected cognitive data diverged between those that would be diagnosed with dementia and healthy controls. To this end, we subdivided group 2 (dementia 1-10) into two groups: those clinically diagnosed within $1-5$ years $(n=133)$ and those clinically diagnosed 6-10 years $(n=147)$ after testing. We then used ANOVA to compare these two subgroups to the healthy controls and to group 1 (dementia 11-22) with regard to education- and age-corrected performance on the tests that significantly predicted dementia diagnosis (SPTB, VTB, and PROSP).

The results showed that age- and education-corrected episodic free recall of sentences with and without enactment (VTB and SPTB) were significantly lower in those that would become demented within 6-10 years and within 1-5 years than in healthy controls (fig. 1). Furthermore, SPTB performance also significantly differentiated between the two subgroups $(p<0.0005)$, and the impact of VTB approached significance $(p=0.061)$. Performance on the 
Boraxbekk et al.: Free Recall Episodic Memory Performance Predicts Dementia Ten

Years prior to Clinical Diagnosis: Findings from the Betula Longitudinal Study

prospective memory task PROSP was lower in the subgroup of individuals that would be clinically diagnosed with dementia within 1-5 years than in all other groups. As expected, we detected no difference between those who would be demented within 11-22 years and healthy controls.

\section{Discussion}

The present work investigated early cognitive signs of a forthcoming dementia diagnosis using representative large-scale cohort data from the Betula study. Our findings strengthen previous indications that free recall tests reflecting episodic memory performance are affected early in the dementing process. Most importantly, our results show the possibility of detecting these impairments 10 years prior to diagnosis, which is substantially earlier than the previously reported detection about 6 years prior to diagnosis. The present findings also support the suggestion that episodic memory tests may identify at-risk individuals irrespective of the dementia subtype, i.e. $\mathrm{AD}$ and $\mathrm{VaD}$. These data strengthen the large body of research suggesting that $\mathrm{AD}$ and $\mathrm{VaD}$ equally affect cognitive performance even at an early subclinical disease phase $[10,12,29,30]$.

In the present study, we used single-time-point data rather than investigating trajectories of memory decline. Both approaches are important for investigating the association between cognition and dementia. However, in studies investigating the rate of decline, memory performance often reaches plateaus where no change is noticed until the appearance of a sudden decline [31,32]. Thus, it has been argued that data from a single time point may be particularly useful for identifying individuals at risk of dementia prior to clinical diagnosis $[33,34]$. Recent data suggest that VaD involves faster deterioration of cognitive functions than AD [35]. Therefore, it is possible that a different study design could reveal other differences between these two subtypes.

Neuroimaging and CSF studies clearly show that it is possible to predict dementia development, and they have enhanced our understanding of the underlying pathophysiological processes [36]. For example, it has been suggested that biological changes (e.g. hippocampal atrophy and $A \beta$ burden) occur within memory-relevant regions in the medial-temporal lobe up to 15 years prior to the time at which dementia can be clinically diagnosed [37-39]. However, although these techniques are promising for initial evaluations [40], such examinations are usually not accessible to those who are not already identified as at-risk individuals. Moreover, there are as yet no longitudinal studies of participants randomly selected from a population. Instead, the data supporting these techniques have primarily been obtained from studies of participants at an increased genetic risk of dementia (see e.g. Bateman et al. [37]).

Our present findings imply that easily conducted neuropsychological tests - such as tests of free recall episodic memory performance - constitute a reliable marker for identifying individuals at risk of dementia 10 years prior to diagnosis. The main strengths of this study include the large sample size of individuals diagnosed with dementia who were randomly selected from the population and the length of follow-up from initial cognitive testing to diagnosis. The results emphasize that tests of episodic function have differing predictive value at early disease stages, with more demanding tasks apparently being more sensitive to detecting a forthcoming dementia diagnosis. Compared with cued recall, free recall is associated with lower retrieval support, which likely caused the differing predictive values between these two tests. As cognitive tests are accessible to most clinicians, free recall episodic memory testing may be a useful tool for the large-scale identification of individuals in need of further assessment and preventative treatment strategies. One limitation of the present study is that 
information on other biomarkers (e.g. $A \beta$ burden or structural brain imaging) was not available, and thus the exact relationship between cognitive markers and other biomarkers remains to be examined in future studies.

There are several ethical considerations related to the identification of individuals at risk of a future dementia diagnosis, and some authors have raised strong concerns that such methods may lead to overdiagnosis and potentially harm people [41]. There is a clear need to develop an ethical framework for future clinical implementation, including key questions such as whether individuals wish to know whether they are at risk [42]. Additionally, creating a valid interface between cognitive measures and other early biomarkers is complicated by the requirements for proper tests and appropriate cut-offs [43]. Even though treatments are not yet available, the work of operationalizing the criteria, determining appropriate cut-off scores [44, 45], and identifying the specificity and sensitivity of different tests is already important [45]. Understanding the sensitivity of the available tests requires obtaining converging evidence from screening different types of early markers, which would likely reduce the risk of both false-positive and false-negative results in a clinical setting. Old age together with low education may still be the strongest predictor of dementia risk. However, our present results showed that, correcting for age and education, free recall episodic memory predicted dementia 10 years prior to the actual clinical diagnosis. These results must be replicated in other settings in order to increase their generalizability.

Our results only partially supported the ability to identify individuals at risk of dementia up to 22 years prior to diagnosis based on cognitive test performance. The cognitive score on one of the free recall tests, SPTB, could indeed identify individuals who would be clinically diagnosed with dementia between 11 and 22 years after initial cognitive testing, even after controlling for education. Although the OR for this test was close to 1 , suggesting a low predictive value, the SPTB test uses a finely graded scale which instead gives the test a reasonably good predictive value. However, when controlling for age, the test could not predict dementia this far ahead of diagnosis. Hence, the present data do not support the suggestion that cognitive testing may be a reliable marker for dementia up to 22 years prior to its onset.

It has previously been suggested that the transition to dementia involves a global cognitive breakdown affecting multiple cognitive domains [8]. Our present results provide limited support for this hypothesis. Three tests - FLU5M (fluency), BLOCK (visuospatial ability), and the MMSE - significantly predicted dementia up to 10 years prior to diagnosis when controlling for education. However, these tests ceased to be significant when age was added to the model. Hence, based on the present findings, the MMSE, which is a commonly used test in clinical and research settings, does not predict dementia onset over a time period of 10 years. The present data showed that prospective memory testing predicted dementia up to 10 years prior to diagnosis. Prospective measures have previously been suggested as a potential marker for dementia [46], which is rational, since dementia involves components that influence multiple neurocognitive systems [47]. However, the control analysis of age- and education-corrected performance on prospective memory tests did not differ for individuals who would develop dementia compared to healthy controls.

In summary, the present novel results demonstrate that free recall tests of episodic memory measured at a single time point - irrespective of dementia subtype (AD or VaD), age, and educational level - provide a marker for future dementia 10 years prior to its onset. This represents a substantial improvement over previous studies that have only reported such a capability 6 years prior to dementia onset. The present findings are encouraging as they indicate that easily conducted psychometric tests could play an essential role in multilevel preclinical diagnostics. 
Boraxbekk et al.: Free Recall Episodic Memory Performance Predicts Dementia Ten Years prior to Clinical Diagnosis: Findings from the Betula Longitudinal Study

\section{Acknowledgements}

We thank all study participants for taking part in this study. We also thank all our coworkers in the Betula study for assistance during data collection. The Betula Project is supported by a grant from the Swedish Research Council (K2010-61X-21446-01) and by a Wallenberg Scholar grant (to L.N.) from the Knut and Alice Wallenberg Foundation. We also thank Alzheimerfonden, the Västerbotten County Council, and Frimurarstiftelsen.

\section{Disclosure Statement}

The authors have no conflicts of interest to disclose.

\section{References}

1 Hodges JR: Alzheimer's centennial legacy: origins, landmarks and the current status of knowledge concerning cognitive aspects. Brain 2006;129:2811-2822.

- Albert MS: Changes in cognition. Neurobiol Aging 2011;32(suppl 1):S58-S63.

3 Bäckman L, Small BJ, Fratiglioni L: Stability of the preclinical episodic memory deficit in Alzheimer's disease. Brain 2001;124:96-102.

4 Small BJ, Fratiglioni L, Viitanen M, et al: The course of cognitive impairment in preclinical Alzheimer disease: three- and 6-year follow-up of a population-based sample. Arch Neurol 2000;57:839-844.

$\rightarrow 5$ Elias MF, Beiser A, Wolf PA, et al: The preclinical phase of Alzheimer disease: a 22-year prospective study of the Framingham cohort. Arch Neurol 2000;57:808-813.

-6 Bäckman L, Jones S, Berger A-K, et al: Cognitive impairment in preclinical Alzheimer's disease: a meta-analysis. Neuropsychology 2005; 19:520-531.

7 Greene JDW, Hodges JR: Identification of famous faces and famous names in early Alzheimer's disease. Relationship to anterograde episodic and general semantic memory. Brain 1996;119:111-128.

8 Bäckman L, Jones S, Berger A-K, et al: Multiple cognitive deficits during the transition to Alzheimer's disease. J Intern Med 2004;256:195-204.

-9 Fabrigoule C, Rouch I, Taberly A, et al: Cognitive process in preclinical phase of dementia. Brain 1998;121: 135-141.

10 Fahlander K, Wahlin Å, Almkvist O, et al: Cognitive functioning in Alzheimer's disease and vascular dementia: further evidence for similar patterns of deficits. J Clin Exp Neuropsychol 2002;24:734-744.

$\checkmark 11$ Jones S, Laukka EJ, Small BJ, et al: A preclinical phase in vascular dementia: cognitive impairment three years before diagnosis. Dement Geriatr Cogn Disord 2004;18:233-239.

12 Bäckman L, Small BJ: Cognitive deficits in preclinical Alzheimer's disease and vascular dementia: patterns of findings from the Kungsholmen Project. Physiol Behav 2007;92:80-86.

13 Nilsson L-G, Bäckman L, Erngrund K, et al: The Betula prospective cohort study : memory, health, and aging. Neuropsychol Dev Cogn B Aging Neuropsychol Cogn 1997;4:1-32.

14 Nilsson L-G, Adolfsson R, Bäckman L, et al: Betula: a prospective cohort study on memory, health, and aging. Neuropsychol Dev Cogn B Aging Neuropsychol Cogn 2004;11:134-148.

15 American Psychiatric Association: DSM-IV: Diagnostic and Statistical Manual of Mental Disorders, ed 4, techn rev. Washington, American Psychiatric Association, 2000.

16 Mousavi M, Jonsson P, Antti H, et al: Serum metabolic biomarkers of dementia. Dement Geriatr Cogn Disord $2014 ; 4: 252-262$.

17 Nyberg L: A structural equation modeling approach to the multiple memory systems question. J Exp Psychol Learn Mem Cogn 1994;20:485-491.

18 Nyberg L, Maitland SB, Rönnlund M, et al: Selective adult age differences in an age-invariant multifactor model of declarative memory. Psychol Aging 2003;18:149-160.

19 Nilsson L-G, Adolfsson R, Bäckman L, et al: The influence of APOE status on episodic and semantic memory: data from a population-based study. Neuropsychology 2006;20:645-657.

-20 Einstein GO, McDaniel MA: Normal aging and prospective memory. J Exp Psychol Learn Mem Cogn 1990;16: 717-726.

21 Nyberg L, Nilsson L-G, Bäckman L: Recall of actions, sentences and nouns: influences of adult age and passage of time. Acta Psychol (Amst) 1992;49:245-254.

-22 Karlsson T, Bäckman L, Herlitz A, et al: Memory improvement at different stages of Alzheimer's disease. Neuropsychologia 1989;27:737-742.

23 Salthouse TA: Speed mediation of adult age differences in cognition. Dev Psychol 1993;29:722-738.

24 Wechsler D: WAIS-R Manual. New York, Psychological Corporation, 1981. 
Boraxbekk et al.: Free Recall Episodic Memory Performance Predicts Dementia Ten Years prior to Clinical Diagnosis: Findings from the Betula Longitudinal Study

25 Kramer NA, Jarvik L: Assessment of intellectual changes in the elderly; in Raskin A, Jarvik L (eds): Psychiatric Symptoms and Cognitive Loss in the Elderly. Washington, Hemisphere, 1979.

26 Dureman I: SRB:1. Stockholm, Psykologiförlaget, 1960.

27 Folstein MF, Folstein SE, McHugh PR: 'Mini-mental state'. A practical method for grading the cognitive state of patients for the clinician. J Psychiatr Res 1975;12:189-198.

28 Ngandu T, von Strauss E, Helkala E-L, et al: Education and dementia: what lies behind the association? Neurology 2007;69:1442-1450.

29 Ott A, Bretler M, Vanharskamp F, et al: Prevalence of Alzheimer's disease and vascular dementia: association with education. The Rotterdam study. BMJ 1995;310:970-973.

-30 Laukka EJ, Jones S, Small BJ, et al: Similar patterns of cognitive deficits in the preclinical phases of vascular dementia and Alzheimer's disease. J Int Neuropsychol Soc 2004;10:382-391.

-31 Rubin EH, Storandt M, Miller JP, et al: A prospective study of cognitive function and onset of dementia in cognitively healthy elders. Arch Neurol 1998;55:395-401.

-32 Smith GE, Pankratz VS, Negash S, et al: A plateau in pre-Alzheimer memory decline. Evidence for compensatory mechanisms? Neurology 2007;69:133-139.

-33 Cerhan JH, Ivnik RJ, Smith GE, et al: Alzheimer's disease patients' cognitive status and course years prior to symptom recognition. Neuropsychol Dev Cogn B Aging Neuropsychol Cogn 2007;14:227-235.

-34 Ivnik RJ, Smith GE, Petersen RC, et al: Diagnostic accuracy of four approaches to interpreting neuropsychological test data. Neuropsychology 2000;14:163-177.

-35 Laukka EJ, Macdonald SWS, Fratiglioni L, et al: Preclinical cognitive trajectories differ for Alzheimer's disease and vascular dementia. J Int Neuropsychol Soc 2012;18:191-199.

-36 Román G, Pascual B: Contribution of neuroimaging to the diagnosis of Alzheimer's disease and vascular dementia. Arch Med Res 2012;43:671-676.

-37 Bateman RJ, Xiong C, Benzinger TL, et al: Clinical and biomarker changes in dominantly inherited Alzheimer's disease. N Engl J Med 2012;367:795-804.

38 Fotuhi M, Do D, Jack C: Modifiable factors that alter the size of the hippocampus with ageing. Nat Rev Neurol 2012;8:189-202.

39 Fox NC, Warrington EK, Freeborough PA, et al: Presymptomatic hippocampal atrophy in Alzheimer's disease. A longitudinal MRI study. Brain 1996;119:2001-2007.

40 Knopman DS, Dekosky ST, Cummings JL, et al: Practice parameter: diagnosis of dementia (an evidence-based review). Report of the Quality Standards Subcommittee of the American Academy of Neurology. Neurology 2001;56:1143-1153.

41 Le Couteur DG, Doust J, Brayne C: Political drive to screen for pre-dementia: not evidence based and ignores the harms of diagnosis. BMJ 2013;347:1-6.

42 Schicktanz S, Schweda M, Ballenger JF, et al: Before it is too late: professional responsibilities in late-onset Alzheimer's research and pre-symptomatic prediction. Front Hum Neurosci 2014;8:921.

-43 Lazarczyk MJ, Hof PR, Bouras C, et al: Preclinical Alzheimer disease: identification of cases at risk among cognitively intact older individuals. BMC Med 2012;10:1-13.

44 Bastin C, Salmon E: Early neuropsychological detection of Alzheimer's disease. Eur J Clin Nutr 2014;68:11921199.

45 Gainotti G, Quaranta D, Vita MG, et al: Neuropsychological predictors of conversion from mild cognitive impairment to Alzheimer's disease. J Alzheimers Dis 2014;38:481-495.

46 Van den Berg E, Kant N, Postma A: Remember to buy milk on the way home! A meta-analytic review of prospective memory in mild cognitive impairment and dementia. J Int Neuropsychol Soc 2012;18:706-716.

-47 Burgess PW, Quayle A, Frith CD: Brain regions involved in prospective memory as determined by positron emission tomography. Neuropsychologia 2001;39:545-555. 